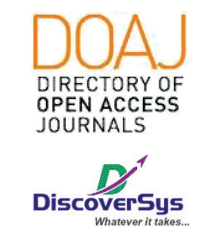

Published by DiscoverSys

\section{Chronic pulmonary aspergillosis - chronic cavitary pulmonary aspergillosis: A case report}

\author{
Francis Celeste, ${ }^{1 *}$ Ency Eveline ${ }^{2}$
}

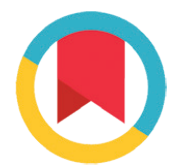

CrossMark

\title{
ABSTRACT
}

Background: Chronic pulmonary aspergillosis (CPA) includes several disease manifestations. Almost all cases of CPA are caused by $A$. fumigatus. There are several underlying diseases that predispose patients to CPA. Treatment is often individualised depending on underlying disease process and the patient's pulmonary status.

Case presentation: A 57-year-old male with a history of renal transplant in the year 2006, routine on immunosuppressants, pulmonary tuberculosis relapse on anti-tuberculosis medications, aspergillosis on long term voriconazole, and DM type 2 presented with dyspnea, massive hemoptysis and productive cough 3 months before admission. Patient was diagnosed with aspergillosis in 0ctober 2012 through bronchoscopy. Microbiology result showed Aspergillus flavus. Enhanced thoracal CT result showed a cavitating nodule with soft tissue lesion in upper right lung, with fibrotic changes in the right lung and mild tubular bronchiectasis, with bilateral pleural thickening. Patient was then planned for lung resection due to the persistent pulmonary cavity. However, his clinical condition worsened and the patient passed away a few days before surgery.

Conclusion: Diagnosing chronic pulmonary aspergillosis can often be challenging. The diagnosis of CPA can be inferred from a single chest radiograph. Despite this, detailed and sequentially acquired radiographic data may be required to observe both the typical radiographic features and the very slow progression of this disease. Treatment is often individualised. Azoles are the antifungal drug of choice when required.
${ }^{1}$ General Practitioner Siloam Hospitals Kebon Jeruk, JakartaIndonesia

${ }^{2}$ General Practitioner, Mochtar Riady Comprehensive Cancer Center (MRCCC), Jakarta-Indonesia
*Correspondence to:

Francis Celeste, General Practitioner Siloam Hospitals Kebon Jeruk, Jakarta-Indonesia

francisceleste3@outlook.com

Received: 2019-09-12 Accepted: 2020-07-10 Published: 2020-08-01

Keywords: chronic pulmonary aspergillosis, individualised treatment, azoles.

Cite This Article: Celeste, F., Eveline, E. 2020. Chronic pulmonary aspergillosis - chronic cavitary pulmonary aspergillosis: A case report. Intisari Sains Medis 11(2): 481-483. D0I: 10.15562/ism.v11i2.614

\section{INTRODUCTION}

Chronic pulmonary aspergillosis (CPA) includes several disease manifestations, including Aspergilloma, Aspergillus nodules, Chronic Cavitary Pulmonary Aspergillosis, and Chronic Fibrosing Pulmonary Aspergillosis. Almost all cases of CPA are caused by A. fumigatus, A. niger, or A flavus. There are several underlying diseases that predispose patients to CPA include pulmonary tuberculosis, nontuberculous mycobacterial infection, allergic bronchopulmonary aspergillosis, asthma, lung cancer, prior pneumothorax with associated bulla formation, chronic obstructive pulmonary disease, and fibro cavitary sarcoidosis. ${ }^{1}$ CPA occurs in patients with chronic cavitary lung disease and is characterised by an indolent clinical course evolving over months to years. ${ }^{2}$

\section{CASE PRESENTATION}

A 57 year old male with a history of renal transplant in the year 2006, routine on immunosuppressants mycophenolate mofetil $500 \mathrm{mg}$ twice daily and cyclosporine $75 \mathrm{mg}$ - 0 - $50 \mathrm{mg}$, pulmonary tuberculosis relapse on anti-tuberculosis medications, aspergillosis on long term voriconazole, and DM type 2 presented with dyspnea, massive hemoptysis and productive cough 3 months prior to admission. Patient is currently on anti-tuberculosis medication for approximately 8 months. Patient brought with him prior examination results during admission, including plain chest X-rays (Figure 1) which exhibited a cavity with intra lesion heterogenous

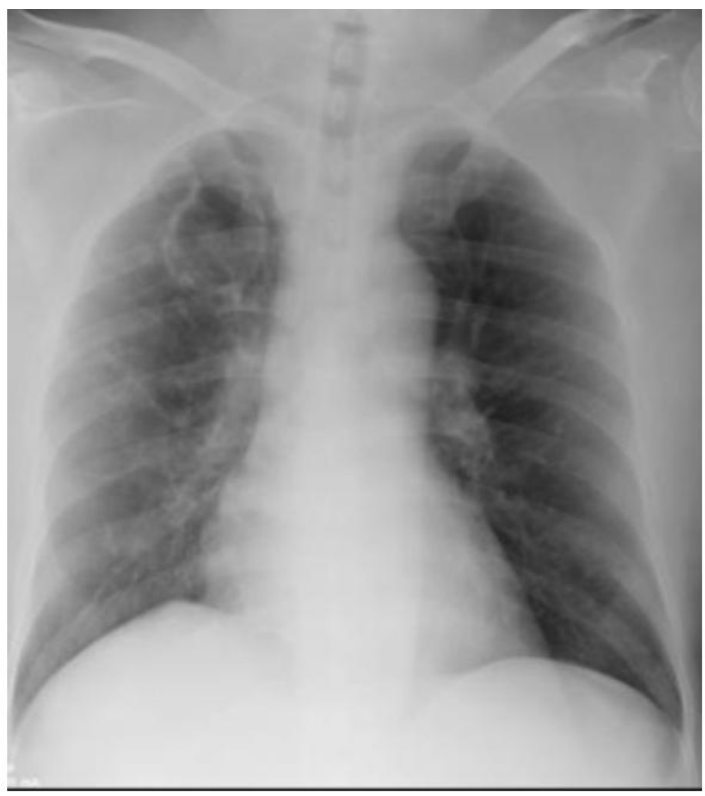

Figure 1 Thorax AP in the year 2015 

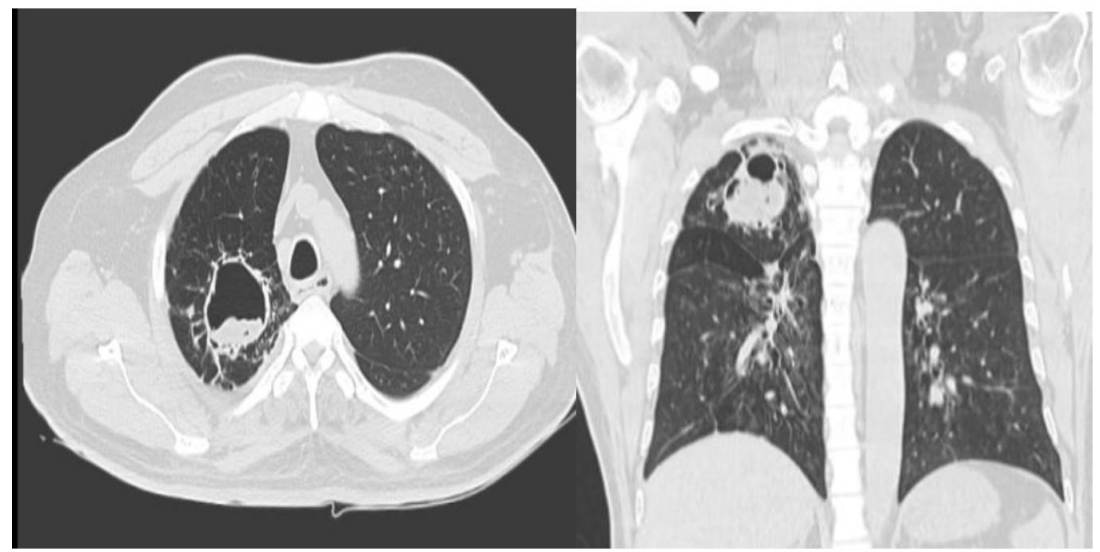

Figure 2 Thoracal CT scan in the year 2015

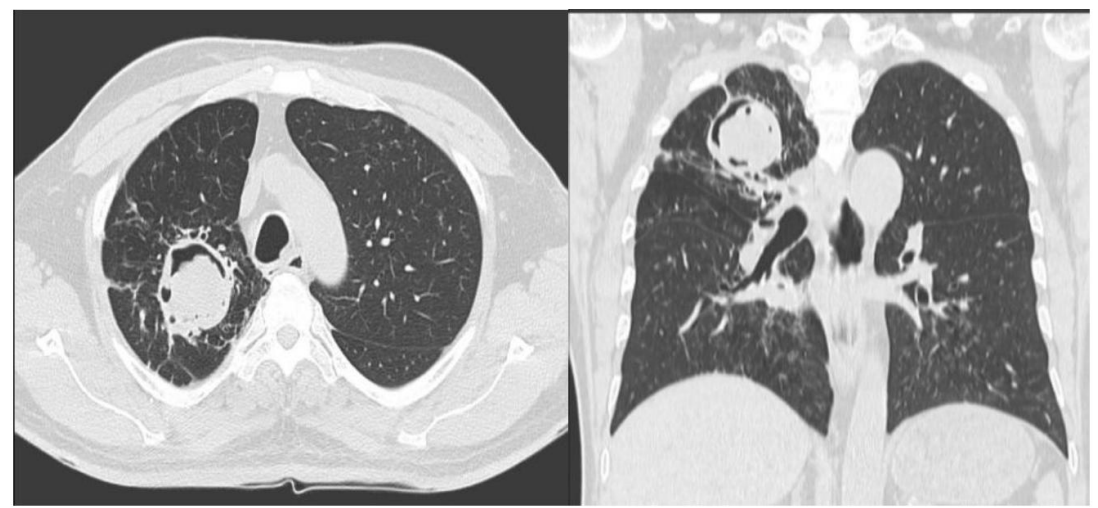

Figure 3 Thoracal CT scan in the year 2017

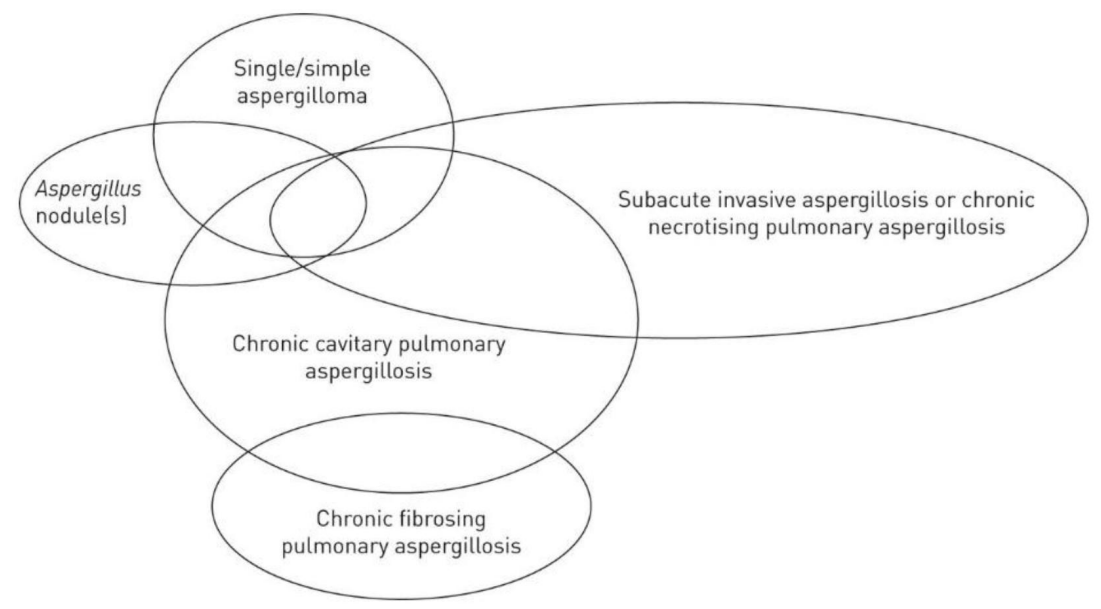

Figure 4 The various conditions classified under CPA3

opacity situated in the right lung apex, suggesting a fungus ball.

An enhanced thoracal CT scan was done and resulted in a cavitating nodule with a soft tissue lesion in the right upper lung segment, measuring approximately $5.36 \times 5.07 \times 4.86 \mathrm{~cm}$, suggesting aspergilloma (Figure 2). Bronchoscopy was then performed, with the microbiology examination showing Aspergillus flavus.

The patient was treated with voriconazole for 1 year and enhanced thoracal CT scan evaluation was performed. Result showed a cavitating nodule with soft tissue lesion in upper right lung, measuring $4.6 \times 3.7 \times 4.2 \mathrm{~cm}$ with fibrotic changes in the right lung and mild tubular bronchiectasis, with bilateral pleural thickening (Figure 3). Patient was then planned for lung resection due to the persistent pulmonary cavity. However, his clinical condition worsened and the patient passed away a few days before surgery.

\section{DISCUSSION}

Chronic pulmonary aspergillosis (CPA) is an uncommon pulmonary disease, often complicating many other respiratory disorders. The most common form of CPA is chronic cavitary pulmonary aspergillosis (CCPA). ${ }^{3} \mathrm{~A}$ duration greater than three months distinguishes chronic pulmonary aspergillosis from acute and subacute invasive pulmonary aspergillosis. ${ }^{1}$ There is an overlap between the various conditions classified under CPA (Figure 4).

Pathogenesis of chronic pulmonary aspergillosis remains incompletely understood. Patients with chronic pulmonary aspergillosis are generally immunocompetent, all having either prior pulmonary damage or disease. ${ }^{1}$ Mucociliary clearance associated with structural lung disease appears to be a critical factor in the pathogenesis of CPA. A history of mycobacterial lung infection, emphysema, COPD, asthma, sarcoidosis, lung cancer, thoracic surgery, marijuana use, and a history of Legionella infection have been described as predisposing conditions for CPA. ${ }^{2}$ There is Increasing evidence that supports genetic factors play a role in patients with chronic cavitary pulmonary aspergillosis. ${ }^{1}$ The Majority of cases of CPA are caused by $A$. fumigatus although there have been cases described with $A$. niger, or $A$ flavus. The age of patients with chronic pulmonary aspergillosis vary from 20 to over 80 years old but are typically in their middle years. ${ }^{1}$

Diagnosing CPA with certainty can be challenging given the presentation has similarities with other chronic respiratory illnesses. Patients can present with a variety of respiratory symptoms. Most commonly are shortness of breath, chronic cough, sputum production, chest discomfort, weight loss and fatigue. Also present in CPA is life-threatening hemoptysis (>150 ml/day) which is a major cause of morbidity and mortality. ${ }^{4}$

The diagnostic criteria of CCPA include one or more pulmonary cavities (with either a thin or thick wall) possibly containing one or more aspergillomas or irregular intraluminal material, with serological or microbiological evidence implicating Aspergillus spp. with significant pulmonary and/or 
systemic symptoms and overt radiological progression (new cavities, increasing pericavitary infiltrates or increasing fibrosis) over at least 3 months of observation. ${ }^{3}$ Other sources cite the need of Aspergillus IgG antibodies being present for the diagnosis of CCPA. ${ }^{1}$

Due to chronic pulmonary aspergillosis representing a spectrum of disease, therapy is often individualised. This is because the risks and benefits of medical and surgical therapy vary with the manifestations of disease and the patient's pulmonary status.

Most patients with CCPA require antifungal therapy. In certain cases where the patient is asymptomatic with radiographically and serologically stable disease, as determined over many months, antifungal treatment is not needed. The azoles, specifically voriconazole and itraconazole, are the main antifungal agents used. Guidelines state that a course of 6 months is given to patients with CCPA. In cases where there is disease progression or immunosuppression, long term antifungal therapy may be necessary. Embolisation may be performed to prevent or treat hemoptysis. Surgical outcomes are not as favourable as those with single aspergillomas. ${ }^{5}$

\section{CONCLUSION}

Chronic pulmonary aspergillosis and its associated disease represents an uncommon pulmonary disease, often complicating many other respiratory disorders. A history of prior lung disease predisposes patients to CPA, where Aspergillosis plays a significant role. The symptoms of CPA can mimic another pulmonary disease hence diligent and detailed follow up are required. The mainstay to therapy of CCPA is antifungal therapy, where 6 months is the minimum required duration. Longer regiments are necessary in ongoing immunosuppression or disease progression.

\section{CONFLICT OF INTEREST}

The author declare there is no conflict of interest regarding publication of current report.

\section{REFERENCES}

1. Graham KG, Nasir A. Chronic cavitary pulmonary aspergillosis: a case report and review of the literature. Am J Case Rep. 2019;20:1220-1224.

2. Izumikawa K. Recent advance in chronic pulmonary aspergillosis. Respir Investig. 2016;54(2):85-91.

3. Denning D, Cadranel J, Beigelman-Aubry C, Ader F, Chakrabarti A, Blot S, Ullmann A, Dimopoulos G. and Lange C. Chronic pulmonary aspergillosis: rationale and clinical guidelines for diagnosis and management. Eur Respir J. 2016;47(1):45-68.

4. Maghrabi F, Denning DW. The Management of Chronic Pulmonary Aspergillosis: The UK National Aspergillosis Centre Approach. Curr Fungal Infect Rep. 2017;11(4):242-251.

5. Kosmidis C, Denning W. The clinical spectrum of pulmonary aspergillosis. Thorax. 2015;70(3):270-7.

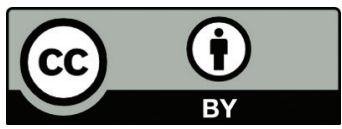

This work is licensed under a Creative Commons Attribution 